phosphatase, and amylase were noted. (Ogunmekan AO, Hwang PA. A randomized, double-blind, placebo-controlled, clinical trial of D-a-tocopheryl acetate (Vitamin E), as add-on therapy, for epilepsy in children. Epilepsia Jan/Feb 1989; 30:84-89).

COMMENT. These authors and others have reported reduced plasma levels of Vitamin $E$ in children taking antiepileptic drugs. Hyperbaric oxygen-induced seizures in rats are prevented by prior administration of Vitamin $E$. (Jerrett SA et al. Aerospace Med 1973;44:40-4). The clinical trial reported here and a previous uncontrolled study support the experimental findings in animals that Vitamin $E$ may inhibit the effects of oxidation in brain tissue and act as a membrane stabilizer in epileptic cerebral cortex. Further trials of this adjunctive treatment for refractory epilepsies are certainly warranted.

\title{
HEADACHE
}

\section{PSYCHOLOGICAL FACTORS IN ADOLESCENT HEADACHE}

Seventy high school students between 16 and 18 years of age reporting $a$ headache frequency of once a week or more were compared with a headache-free control group and were studied by questionnaires for psychosocial, health-behavior, and medical problems at the Dept of Child and Youth Psychiatry, University Hospital of Uppsala, Sweden. Adolescents with recurrent tension and migraine headaches reported significantly more somatic symptoms and psychological distress than controls, they were more often absent from school, and used the school health service more than controls. Their parents were more often divorced and suffered more frequently from headache and abdominal pain. Nervous problems, anxiety, depression, homework time, somatic symptoms and absence from school were psychosocial predictors of headache susceptibility. (Larsson B. The role of psychological, health-behavior and medical factors in adolescent headache. Dev Med Child Neurol Oct 1988; 30:616-625).

COMMENT. These results differ from a previous study of anxiety in childhood migraine. Patients with migraine and their parents who completed standardized anxiety, personality, and life-event scales showed no significant difference from controls. All patients had anxiety scores within normal. Patient selection and the omission of tension headache sufferers could explain the difference in findings.

\section{RELAXATION TREATMENT FOR MIGRAINE}

Relaxation training was compared to two control placebo psychological methods of treatment in 99 children and adolescents with frequent migraine at the Children's Hospital of Eastern Ontario, University of Ottawa, Canada. Relaxation methods consisted of 6 , one-hour, weekly sessions in which children were taught sequential tensing and relaxation of large muscle groups and the use of deep breathing. Placebo treatment consisted of therapy sessions to teach recognition of emotions, 